\title{
Compact ultra-wide band microwave filter utilizing quarter-wave length short-circuited stubs with reduced number of vias
}

\begin{abstract}
We present here a novel pattern with compact size of ultra-wide band microstrip filter. The filter is originally modeled from five poles quarter-wavelength short-circuited stubs. It is then, transformed into a new compact butterfly-like pattern. This new "butterflyshaped" pattern consists of three vias instead of five vias from its original design. The prototype has shown an improvement in scattering parameters measurement compared with its original model. It delivers $112 \%$ of fractional bandwidth, magnitude of insertion loss better than $1.6 \mathrm{~dB}$ and return loss lower than $-8 \mathrm{~dB}$. It is fabricated on RT Duroid 5880 with 2.2 dielectric constant, $0.508 \mathrm{~mm}$ of substrate thickness and $35 \mu \mathrm{m}$ copper thickness. The overall dimension of the filter is $20.7 \times 15.8 \mathrm{~mm} 2$.
\end{abstract}

Keyword: Compact; Microstrip; Microwave filter; Short-circuited stubs; Ultra-wide band 